\title{
Effects of Anxiety on Canine Movement in Dog-Robot Interactions
}

\author{
Anna Zamansky ${ }^{1 *}$, Stephane Bleuer-Elsner, ${ }^{1,2}$, Sylvia Masson $^{3}$, Shir Amir ${ }^{1}$, Ofer Magen ${ }^{1}$, \\ and Dirk van der Linden ${ }^{4}$
}

\author{
${ }^{1}$ University of Haifa, Haifa, Israel \\ ${ }^{2}$ Private Veterinary Practice, Tel Aviv, Israel \\ ${ }^{3}$ Private Veterinary Practice, Voreppe, France \\ ${ }^{4}$ University of Bristol, UK \\ *Corresponding author (Email: annazam@is.haifa.ac.il)
}

Citation - Zamansky, A., Bleuer-Elsner, S., Masson, S., Amir, S., Magen, O., \& van der Linden, D. (2018). Effects of anxiety on canine movement in dog-robot interactions. Animal Behavior and Cognition, 5(4), 380-387. https://doi.org/10.26451/abc.05.04.05.2018

\begin{abstract}
Canine behavioral disorders, such as various forms of fear and anxiety, are a major threat for the wellbeing of dogs and their owners. They are also the main cause for dog abandonment and relinquishment to shelters. Timely diagnosis and treatment of such problems is a complex task, requiring extensive behavioral expertise. Accurate classification of pathological behavior requires information on the dog's reactions to environmental stimuli. Such information is typically self-reported by the animal's owner, posing a threat to its accuracy and correctness. Simple robots have been used as controllable stimuli for evoking particular canine behaviors, leading to the increasing interest in dog-robot interactions (DRIs). We explore the use of DRIs as a tool for the assessment of canine behavioral disorders. More concretely, we ask in what ways disorders such as anxiety may be reflected in the way dogs interact with a robot. To this end, we performed an exploratory study, recording DRIs for a group of 20 dogs, consisting of $10 \mathrm{dogs}$ diagnosed by a behavioral expert veterinarian with deprivation syndrome, a form of phobia/anxiety caused by inadequate development conditions, and 10 healthy control dogs. Pathological dogs moved significantly less than the control group during these interactions.
\end{abstract}

Keywords - Dog-robot interactions, DRI, Veterinary behavior, Canine anxiety

Behavioral disorders are a major threat for the well-being of companion dogs and their owners. Moreover, such disorders may lead to dogs' relinquishment to shelters, or prevent shelter dogs from being adopted, eventually leading to their euthanasia (Scarlett, Salman, New, \& Kass, 2002; Winslow, Payne, \& Robles, 2018). Their early diagnosis and treatment are thus of crucial importance. Correct diagnosis and treatment is a complex problem requiring in-depth knowledge of veterinary behavior, a topic that has recently been reported to receive insufficient attention in the standard veterinary curricula (Shivley, Garry, Kogan, \& Grandin, 2016). The classification of pathological disorders and treatment decisions of a behavior specialist veterinarian are usually done on the basis of the reactions of the dog to various environmental stimuli, produced by, among others, the owner, other people, other dogs, and the environment itself. However, such situations are not controllable and not easily recreated during an examination session. Thus, in their diagnosis, behavioral experts mainly rely on owners' self-reporting, the accuracy and correctness of which may be compromised. 
One idea used for producing controllable stimuli for studying animal behavior is using artificial agents (see, e.g., Frohnwieser, Murray, Pike, \& Wilkinson, 2016; Krause, Winfield, \& Deneubourg, 2011). In particular, various robots have been used for studying canine social behaviors. For example, Leaver and Reimchen (2008) investigated the approach preference of dogs towards a dog-like robot with different tail sizes and movements. Gergely, Petró, Topál, and Miklósi (2013) examined dogs' interactive behavior in a problem-solving task in which the dog had no access to the food with three different social partners, two of which were simple robots (remotely controllable cars), and the third - a human behaving in a robot-like manner. Dogs' interactions with more complex commercial robots, displaying a wide variety of (programmed) behavior and/or similarity to the target species, have also been explored (Kubinyi et al., 2004). Given that dogs exhibit social behaviors towards robots, we hypothesized that canine behavioral disorders that are related to social fear, may also be reflected in the way dogs interact with robots.

Fear and anxiety are common behavioral problems that veterinary behavioral experts encounter in their daily practice. Owners of dogs suffering from these problems often report difficulties in walking the dog outside, lack of home training capacity, alertness and panic in the house that may lead to destructions or self-injuries, and sometimes aggressiveness towards humans or other dogs (Tiira, Sulkama, \& Lohi, 2016).

The above issues are often associated with inadequate behavioral development of the puppy, such as a lack of positive exposures during the socialization period (e.g., 3 weeks to 3 months). This may trigger low adaptability capacities in the future and lead to higher risk of developing severe symptoms like fearful and/or aggressive behavior toward unknown humans. In the French Veterinary Psychiatry community $^{1}$ anxious/phobic behavior due to inadequate development is referred to as deprivation syndrome (DS) (Beata, 2006; Mège et al., 2003). This syndrome is assessed based on levels of severity. The lightest one is Deprivation Syndrome Stage 1 (DS1), at which the dog exhibits symptoms of phobia (excessive, long lasting fear) when exposed to an identified fear-inducing stimulus; the signs appear with the stimulus and cease when the stimulus stops. Other stages (Deprivation Syndrome Stage 2 and 3) can be more severe, exhibiting signs of anxiety with inhibition even without an identified stimulus in the dog's environment, and even extreme states such as depression.

In this study, we explore DRIs of dogs exhibiting phobic behaviors in the presence of identified stimuli, focusing specifically on dogs diagnosed with deprivation syndrome, DS1, using the French terminology described above. More specifically, we adapt the experimental paradigm of using DRI for evoking behavioral responses, aiming to investigate the behavior of such dogs through the lens of dogrobot interactions. The idea is that a robot may present a stimulus that is fear-inducing for dogs with deprivation syndrome. As such, they are more bound (than healthy dogs) to respond in a characteristic phobic-like reaction, such as avoidance, flight or fight, freezing in place and moving away from the robot. Our research question is, therefore, do dogs with deprivation syndrome exhibit the same patterns of activity as normal dogs during interactions with a robot? We hypothesized that, due to expected inhibition and avoidance behavior of such dogs, they would be less active in interacting with the robot compared to normal dogs.

\section{Methods}

\section{Subjects}

Twenty dogs were selected for the study. The control group ( $n=10,5$ males, 5 females) had a median age of 3 years ( \pm 2 , max 7, min 8 months), median weight of $23 \mathrm{~kg}( \pm 20$, max 77, min 7), whereas the pathological group ( $n=10,5$ males, 5 females) had a median age of 4 years $( \pm 2$, max 8.5, min 1.5), median weight of $13.5 \mathrm{~kg}( \pm 9, \max 29, \min 2.6)$ (see Table 1 for details).

Table 1

\footnotetext{
${ }^{1}$ https://www.zoopsy.com/
} 
Demographic Information of Selected Dog Subjects

\begin{tabular}{|c|c|c|c|c|c|}
\hline \multicolumn{6}{|c|}{ Control Group $(n=10)$} \\
\hline Name & $\operatorname{Sex}(\mathrm{m} / \mathrm{f})$ & Age (yrs) & Breed & Weight (kgs) & Neutered \\
\hline Gino & $\mathrm{M}$ & 0.8 & Cane Corso & 44 & $\mathrm{n}$ \\
\hline Nancy & $\mathrm{F}$ & 7 & Poodle & 7 & $\mathrm{y}$ \\
\hline Mila & $\mathrm{F}$ & 3.5 & Mixed & 23 & $\mathrm{y}$ \\
\hline Mika & $\mathrm{F}$ & 7 & Mixed & 7 & $\mathrm{y}$ \\
\hline Belle & $\mathrm{F}$ & 4 & Mixed & 23 & $\mathrm{y}$ \\
\hline Ice & M & 2 & Mixed & 28 & $\mathrm{y}$ \\
\hline Theresa & $\mathrm{F}$ & 0.8 & Saluki & 16.5 & $\mathrm{y}$ \\
\hline Pit & M & 1 & Mixed & 25 & $\mathrm{y}$ \\
\hline Jimmy & M & 4.5 & Whippet & 17 & $\mathrm{y}$ \\
\hline Brutus & $\mathrm{M}$ & 2.5 & Bullmastiff & 77 & $\mathrm{n}$ \\
\hline \multicolumn{6}{|c|}{ Pathological Group $(n=10)$} \\
\hline Name & $\operatorname{Sex}(\mathrm{m} / \mathrm{f})$ & Age (yrs) & Breed & Weight (kgs) & Neutered \\
\hline Fufu & $\mathrm{M}$ & 4 & Shitzu & 8 & $\mathrm{y}$ \\
\hline King & M & 2 & Dachshund & 7 & $\mathrm{n}$ \\
\hline Louis & M & 1.5 & Mixed & 25 & $\mathrm{n}$ \\
\hline Lois & $\mathrm{F}$ & 8 & Mixed & 29 & $\mathrm{y}$ \\
\hline Luis & $\mathrm{F}$ & 4 & Mixed & 14 & $\mathrm{y}$ \\
\hline Milki & M & 1.5 & Shitzu & 5.5 & $\mathrm{y}$ \\
\hline Oliver & M & 5 & Maltese & 2.6 & $\mathrm{y}$ \\
\hline Pie & $\mathrm{F}$ & 4 & Mixed & 20 & $\mathrm{y}$ \\
\hline Kuka & $\mathrm{F}$ & 8.5 & Mixed & 13 & $\mathrm{y}$ \\
\hline Goldy & $\mathrm{F}$ & 3 & Mixed & 21 & $\mathrm{y}$ \\
\hline
\end{tabular}

All subjects in the pathological group were clinical patients of two behavioral veterinary clinics. The DRI was recorded at the time of their first visit of a behavioral expert veterinarian, who used French diagnostic approach according to (Beata, 2006; Mège et al., 2003; Pageat, 1998). The inclusion criteria for the pathological group were the following:

1. This was the subject's first visit to the behavioral expert veterinarian.

2. During the visit, the expert diagnosed the subject with deprivation syndrome (stage 1, DS1).

3. During the visit, the expert prescribed a medical treatment for the above condition.

To further ensure the correctness of expert diagnosis, for each of the included participants, we made sure that a follow-up visit took place several weeks later, at which the owner reported improvement of the dog's condition as a result of taking the prescribed medication.

All subjects in the control group were dogs with no reported behavioral problems or issues. They were recruited in the two clinics during standard checkup and vaccination, or through the authors' professional connections.

\section{The Robot}

We used a simple commercial dog-shaped toy robot of size $10 \mathrm{~cm} \times 14 \mathrm{~cm} \times 6 \mathrm{~cm}$ (see Figure 1), which made repeated circular movements and barking noise. The latter was disabled by removing the robot's vocalization mechanism. The robot was placed in a fixed location (marked by $\mathrm{X}$ in Figure 1) during veterinary examination. 


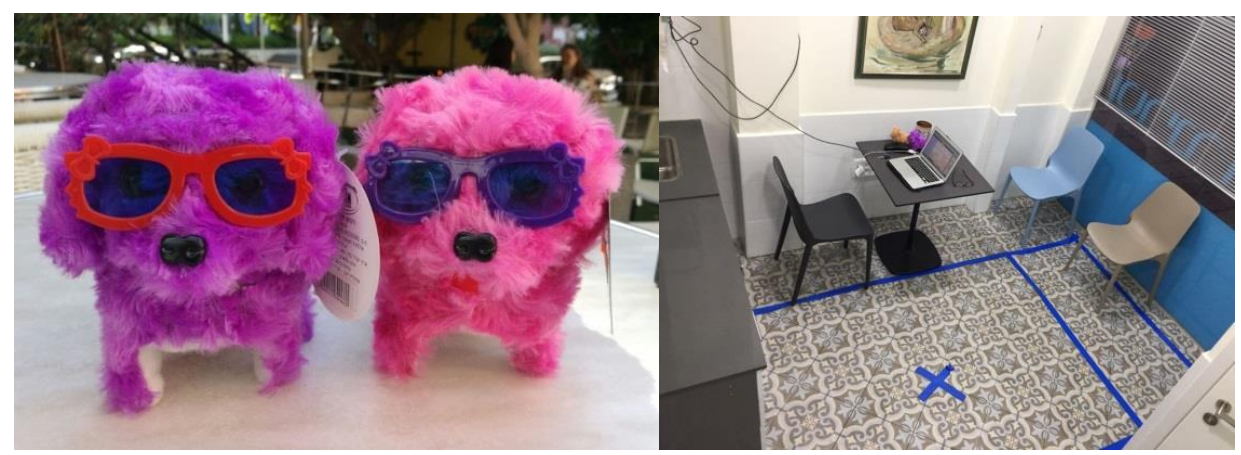

Figure 1. Example of the type of robot used and the experimental setup.

\section{Location}

Dogs were observed in the veterinary behavioral clinics at the Petah Tikva and Tel Aviv Animal Hospitals. The testing space captured by a camera fixed on the ceiling was $100 \times 65 \mathrm{~cm}$ and $300 \times 160$ $\mathrm{cm}$ respectively (see Figures 1 and 2).

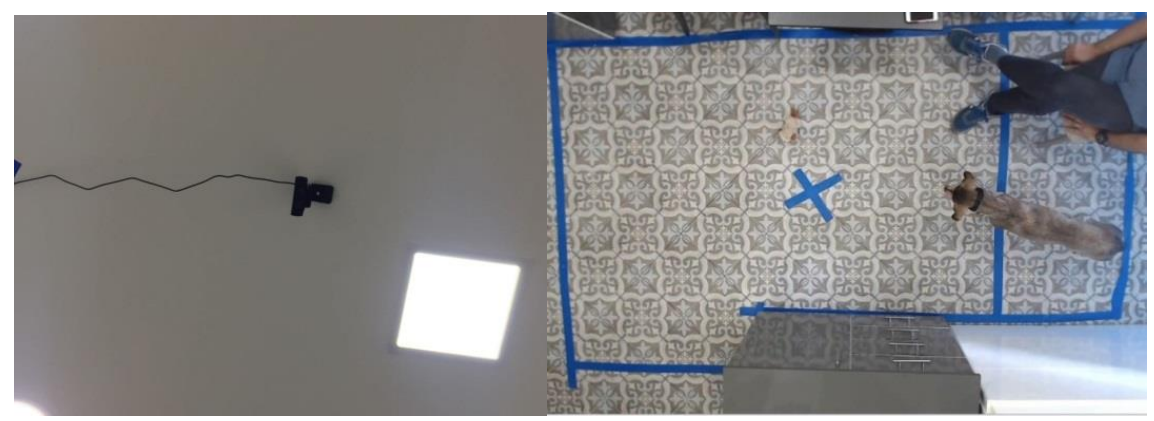

Figure 2. Camera fixed on the ceiling in Tel Aviv clinic and an example frame.

\section{Procedure}

The moving robot was introduced as part of a behavioral consultation session lasting 40-50 min. Each session was structured according to the protocol detailed below and recorded using a stationary video-camera placed on the ceiling. The veterinary doctor and the owner(s) were always placed in the same location (fixed chairs in the room), excepting the moment at which the doctor introduced the robot in the middle of the room. An owner with his dog is shown in Figure 3.

Each session consisted of three phases as described below.

Preliminary phase. The dog was brought into the room and taken off its leash. The owner(s) took their place at a predefined spot in the room. They were requested not to interact or make eye contact with the dog during the experiment, regardless of what the dog was doing. Video recording was begun. The dog was allowed to freely move around the room while the veterinarian interviewed the owner(s) and also filled out information on his computer.

Introduction phase. About 20 min into the interview, the veterinarian placed the inactive robot in the center of the room and returned to his chair. The dog was recorded for 3 min.

Testing phase. The veterinarian activated the robot and returned to his place. Interaction of the dog with the moving robot was recorded for three minutes. The veterinarian then deactivated the robot and put it away.

The dog was recorded for an additional 10 min after the end of the testing phase. The introduction phase was introduced in order to allow the dog to get acquainted with a strange object, thus preventing very high stress levels of patient dogs. 


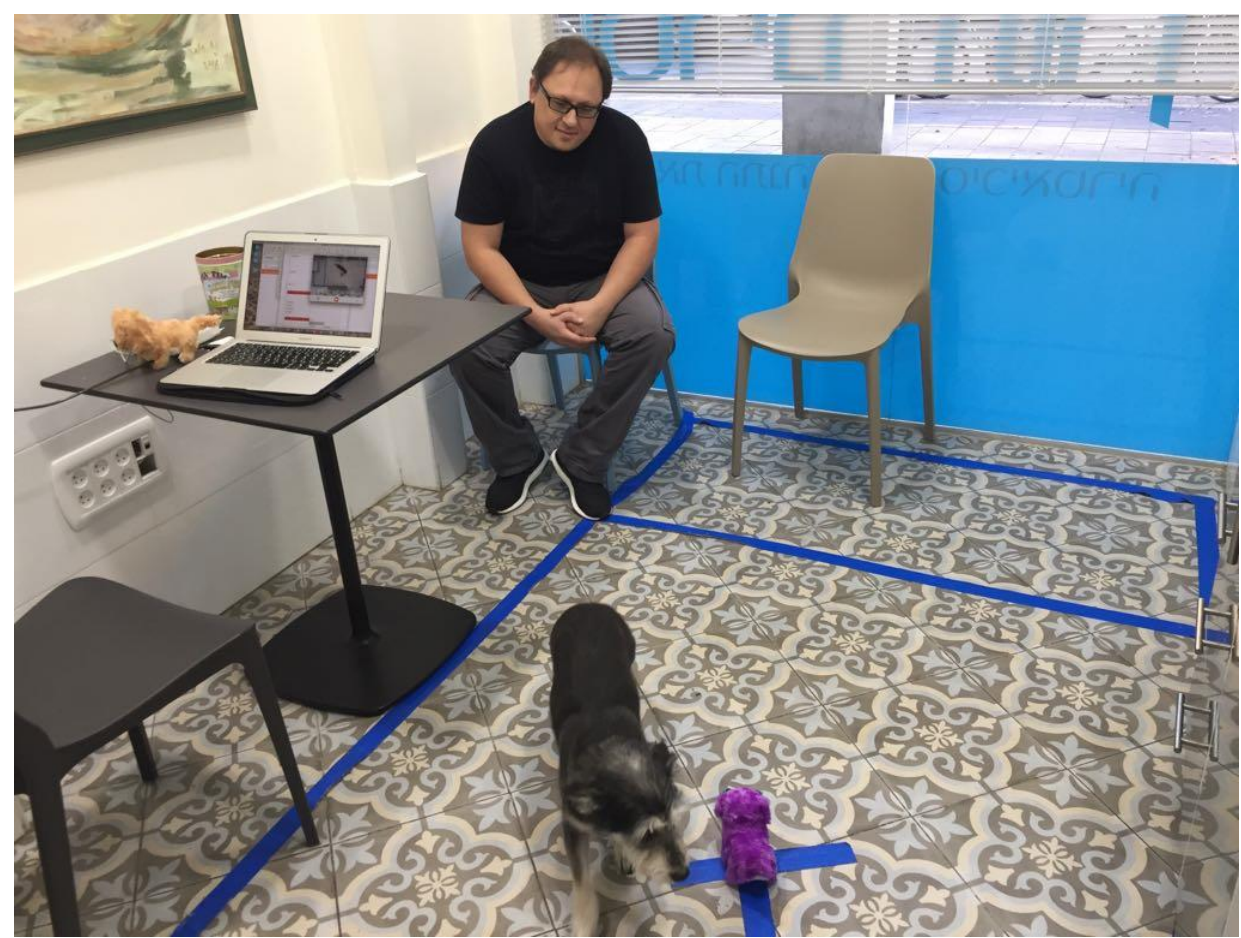

Figure 3. Owner and dog in Tel Aviv clinic.

\section{Data Analysis}

The following variables were selected for data analysis: average speed, maximal speed, average acceleration, maximal acceleration, and percentage of frames (out of total number of frames) where movement was detected. Further details on the selected variables are provided in Table 2.

Table 2

\begin{tabular}{|c|c|c|}
\hline Variable & Explanation & Unit \\
\hline Avg_speed & Average speed of movement & $\mathrm{cm} / \mathrm{s}$ \\
\hline Max_speed & $\begin{array}{l}\text { Maximal speed of movement after smoothing using moving } \\
\text { average with interval of } 10 \text { frames }\end{array}$ & $\mathrm{cm} / \mathrm{s}$ \\
\hline Avg_acc & Average acceleration & $\mathrm{cm} / \mathrm{s}^{2}$ \\
\hline Max_acc & $\begin{array}{l}\text { Maximal acceleration after smoothing using moving } \\
\text { average with interval of } 10 \text { frames }\end{array}$ & $\mathrm{cm} / \mathrm{s}^{2}$ \\
\hline Per_movement & Percentage of frames where movement was detected & \\
\hline
\end{tabular}

We used a self-developed software tool, K9-Blyzer (Amir, Zamansky, \& van der Linden, 2017) for analyzing the video footage recorded at the clinics and producing the data on the movement variables listed in Table 2. Figure 4 shows some example frames where the tool identifies the dog; an example recording can also be found here. For more information on K9-Blyzer, see Amir et al. (2017) and the K9Blyzer website. 


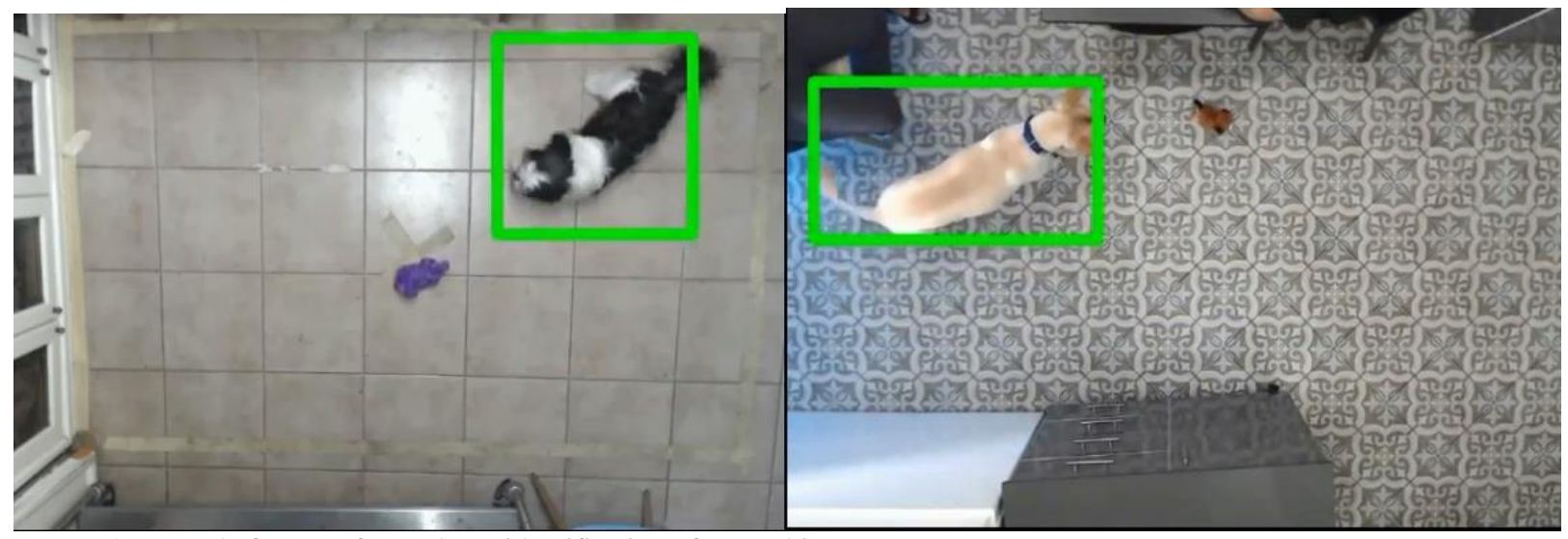

Figure 4. Example frames of K9-Blyzer identification of two subjects.

To the best of our knowledge, this is the first known use of automatic video analysis for canine movement analysis, and, as such, the accuracy of such analysis should be further studied. The accuracy of the detection of some behaviors has been evaluated in Amir et al. (2017). To ensure accuracy of measurement, automatic detection of the dogs in frames was manually validated using the BORIS ethological observation software (Friard \& Gamba, 2016). Frames with incorrect detection by the automatic tool were counted and ranged between $1 \%-10 \%$ for all of the used videos.

\section{Results}

Table 3 shows the median values for the investigated variables for pathological and control groups, and whether they differ significantly. Mann-Whitney U test indicated that Per_movement was significantly lower for pathological anxiety/DS1 group (median $=0.36, S E=0.078$ ) than for control group (median $=0.69, S E=0.046$ ), $U=8, p=.00084$. Thus, the main finding was that pathological dogs were less active than control group dogs during DRI (see Figure 5).

Another (non-significant but notable) finding was that Max_acc was lower for the pathological anxiety/DS1 group (median $=6281.65, S E=4450.81$ ) than for the control group (median $=14650.45, S E$ $=7222.35, U=29, p=.06$, see Figure 5).

Table 3

Observed Values for Analyzed Variables and Differences Between Groups

\begin{tabular}{lccc}
\hline \multicolumn{1}{c}{ Variable } & $\begin{array}{c}\text { Median } \\
\text { (Pathological) }\end{array}$ & $\begin{array}{c}\text { Median } \\
\text { (Control) }\end{array}$ & Mann-Whitney U \\
\hline Avg_speed $(\mathrm{cm} / \mathrm{s})$ & 7.41 & 9.96 & $U=45, p=.73$ \\
Max_speed $(\mathrm{cm} / \mathrm{s})$ & 1161 & 1291.50 & $U=48, p=.91$ \\
Avg_acc $\left(\mathrm{cm} / \mathrm{s}^{2}\right)$ & -8.93 & -7.23 & $U=50, p=.48$ \\
Max_acc $\left(\mathrm{cm} / \mathrm{s}^{2}\right)$ & 6281.65 & 14650.45 & $U=29, p=.06$ \\
Per_movement $(\%)$ & 0.36 & 0.69 & $U=8, \boldsymbol{p}=\mathbf{. 0 0 0 8 4}$ \\
\hline Note. Negative values for Avg acc reflect the dog slowing
\end{tabular}

Note. Negative values for Avg_acc reflect the dog slowing down. 


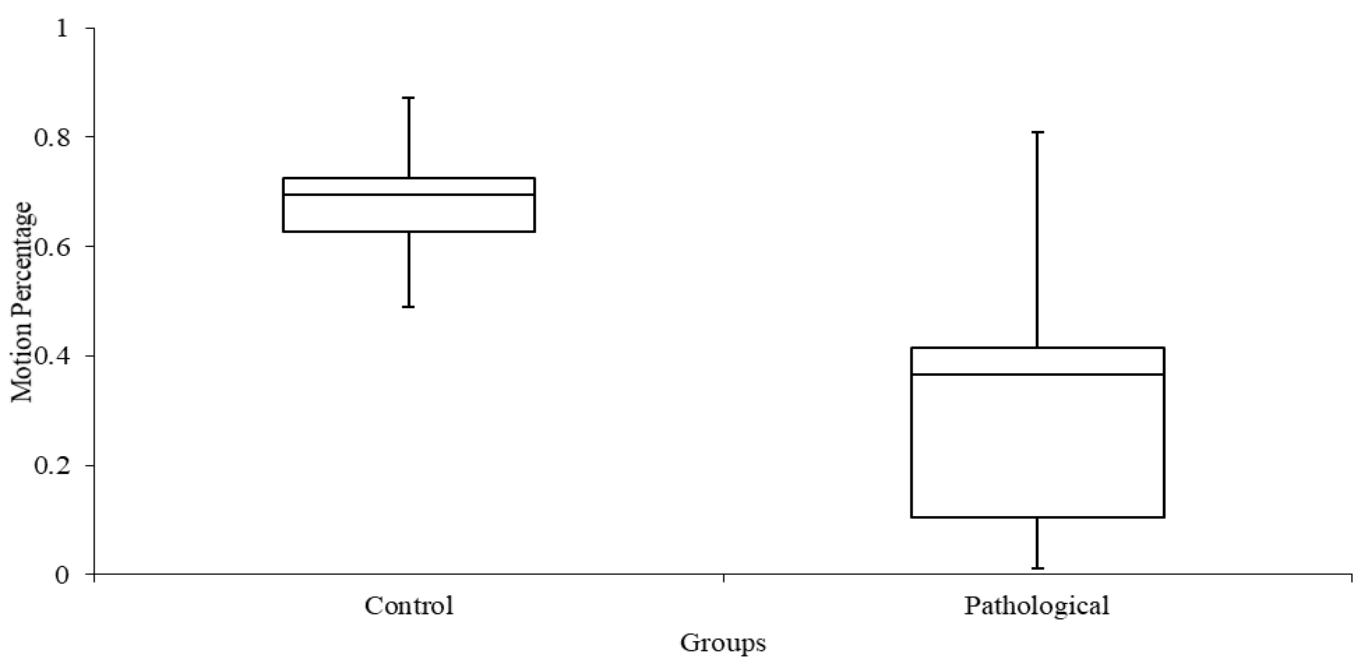

Figure 5. Comparison of motion percentage distribution for control and pathological groups (showing median, $1^{\text {st }}$ and $3^{\text {rd }}$ quartiles, and upper/lower limits).

\section{Discussion}

Pathologically anxious or phobic behavior greatly compromises the welfare of dogs and their owners. According to the French model of zoopsychiatry, the nosography of deprivation syndrome is one of the main pathological expressions of such behavior. Dogs with deprivation syndrome are reported to show avoidance and flight or fight responses, slow and cautious movement dominated by hyper-vigilance when exposed to stimuli inducing fear (Mège et al., 2003). This can explain the result of lesser movement by the pathological group when exposed to a stimulus that may potentially induce fearful reaction, as well as the lower maximal acceleration, indicating less tendency to make rapid movements.

The need for more precise terminology and more quantifiable and objective tools for assessment in veterinary behavior has long been recognized (Overall, Tiira, Broach, \& Bryant, 2014). The French zoopsychiatry model takes a step in this direction, gathering symptoms into pathological entities, such as deprivation syndrome, and associating with them levels of severity. But also within this model there is a lot of room for subjectivity in the evaluation of levels of fears and anxiety, which makes a difference when deciding on diagnosis and on therapy and/or behavioral modification. Therefore, developing quantifiable characteristics of fear-related responses, such as measuring the amount or speed of movement during DRIs, may open up new possibilities for making the assessment of levels of fear and anxiety more objective. Moreover, DRIs protocols such as the one used in our study are very easy to reproduce in a standard consultation room: all they need is a cheap and easily available commercial robotic toy. In conjunction with automatic tools for online movement analysis, such procedures have the potential to provide just-in-time support for decision making of behavioral veterinary experts, as well as general veterinarians.

In the current study, we have employed an automatic tool, which can complement (and even outperform) human analysis in two main aspects. First, the nature of the measured variables highlights the potential of automatic analysis of behavior. Namely, motion percentage, speed or acceleration are very hard to establish by looking at a dog during a veterinary session, or even in a systematic manual video analysis. Secondly, the ability to process, in real time, large volumes of data makes automatic tools particularly appealing in the context of real-time decision support, or for comparing over time the effect of medical treatment a dog receives for treating behavioral disorders. These two aspects highlight the potential of using dog-robot interactions, coupled with automatic tools, to support decision making in veterinary settings. It could also be useful in the context of behavioral evaluations (e.g., working dogs, shelter dogs, etc.). 


\section{Summary and Future Research}

Our findings highlight the potential of using DRI for gaining better understanding of the behavior of dogs afflicted with various behavioral disorders. Future research in this direction could provide the basis for enhancing and guiding veterinary protocols of diagnosing behavioral disorders. Phobia is only one of many behavioral disorders that can be explored in this setting. Other examples include hyperactivity or ADHD-like behaviors. Hyperactive dogs are known to express their pathology by a level of motion characterized by high speed and lack of coherence (Marlois \& Beata 2016; Puurunen, Tiira, Lehtonen, Hanhineva, \& Lohi, 2016). In dogs with separation anxiety, on the other hand, the quality of attachment between the dog and their owner may be explored in a way similar to the one used in this study measuring variables such as distance from the owner, distance from the veterinarian, and search of the owner proximity when a stimulation occurs in the environment (Houpt, Honig, \& Reiser, 1996).

\section{References}

Amir, S., Zamansky, A., \& van der Linden, D. (2017, November). K9-Blyzer: Towards video-based automatic analysis of canine behavior. In Proceedings of the Third International Animal-Computer Interaction Conference (p. 9). ACM.

Beata, C. (2006). Terminology think tank: Terminology, a European view. Journal of Veterinary Behavior: Clinical Applications and Research, $1,80-83$.

Friard, O., \& Gamba, M. (2016). BORIS: A free, versatile open-source event-logging software for video/audio coding and live observations. Methods in Ecology and Evolution, 7, 1325-1330.

Frohnwieser, A., Murray, J. C., Pike, T. W., \& Wilkinson, A. (2016). Using robots to understand animal cognition. Journal of the Experimental Analysis of Behavior, 105, 14-22.

Gergely, A., Petró, E., Topál, J., \& Miklósi, Á. (2013). What are you or who are you? The emergence of social interaction between dog and an unidentified moving object (UMO). PloS One, 8, e72727.

Houpt, K. A., Honig, S. U., \& Reisner, I. R. (1996). Breaking the human-companion animal bond. Journal of the American Veterinary Medical Association, 208, 1653-1659.

Krause, J., Winfield, A. F., \& Deneubourg, J.-L. (2011). Interactive robots in experimental biology. Trends in Ecology \& Evolution, 26, 369-375.

Kubinyi, E., Miklósi, Á., Kaplan, F., Gácsi, M., Topál, J., \& Csányi, V. (2004). Social behaviour of dogs encountering AIBO, an animal-like robot in a neutral and in a feeding situation. Behavioural Processes, 65, 231-239.

Leaver, S. D. A., \& Reimchen, T. E. (2008). Behavioural responses of Canis familiaris to different tail lengths of a remotely-controlled life-size dog replica. Behaviour, 145, 377-390.

Mège, C., Béata, C., Beaumont-Graff, E., Diaz, C., Habran, T., ...Muller, G. (2003). Pathologie comportementale du chien. Masson-AFVAC, Paris, FR.

Overall, K. L., Tiira, K., Broach, D., \& Bryant, D. (2014). Genetics and behavior: A guide for practitioners. Veterinary Clinics: Small Animal Practice, 44, 483-505.

Pageat, P. (1998). Pathologie du comportement du chien (in French). Editions du Point Vétérinaire, Maison565 Alfort. p. 270-274.

Puurunen, J., Tiira, K., Lehtonen, M., Hanhineva, K., \& Lohi, H. (2016). Non-targeted metabolite profiling reveals changes in oxidative stress, tryptophan and lipid metabolisms in fearful dogs. Behavioral and Brain Functions, 12, 7.

Scarlett, J. M., Salman, M. D., New, J. G., \& Kass, P. H. (2002). The role of veterinary practitioners in reducing dog and cat relinquishments and euthanasias. Journal of the American Veterinary Medical Association, 220, 306-311.

Shivley, C. B., Garry, F. B., Kogan, L. R., \& Grandin, T. (2016). Survey of animal welfare, animal behavior, and animal ethics courses in the curricula of AVMA Council on Education-accredited veterinary colleges and schools. Journal of the American Veterinary Medical Association, 248, 1165-1170.

Tiira, K., Sulkama, S., \& Lohi, H. (2016). Prevalence, comorbidity, and behavioral variation in canine anxiety. Journal of Veterinary Behavior: Clinical Applications and Research, 16, 36-44.

Winslow, T., Payne, S. W., \& Robles, K. (2018). Functional analysis and treatment of problem behavior in three animal shelter dogs. Journal of Veterinary Behavior, 26, 27-37. 